\title{
A 26-year-old Woman With Bilateral Leg Pain and Pruritus
}

\author{
S. M. Morell MD $₫$, Jerad M. Gardner MD, L. J. Suva PhD, \\ C. O. Montgomery MD
}

Received: 10 April 2015/Accepted: 8 July 2015/Published online: 17 July 2015

(C) The Association of Bone and Joint Surgeons(B) 2015

\section{History and Physical Examination}

A 26-year-old woman with an extensive medical history significant for Crohn's disease, autoimmune hepatitis, primary sclerosing cholangitis, idiopathic thrombocytopenic purpura, and abdominal lymphadenopathy presented with a chief complaint of chronic bilateral leg pain. The patient also had a long-standing history of fatigue, abdominal pain, and pruritus for several years.

Before presentation, she had CT of the abdomen for abdominal pain. This study revealed diffuse lymphadenopathy throughout the abdomen with the largest

Each author certifies that he or she, or a member of his or her immediate family, has no funding or commercial associations (eg, consultancies, stock ownership, equity interest, patent/licensing arrangements, etc) that might pose a conflict of interest in connection with the submitted article.

All ICMJE Conflict of Interest Forms for authors and Clinical Orthopaedics and Related Research ${ }^{\mathbb{R}}$ editors and board members are on file with the publication and can be viewed on request.

Each author certifies that his or her institution approved the reporting of this case report, that all investigations were conducted in conformity with ethical principles of research, and that informed consent for participation in the study was obtained.

S. M. Morell ( $₫)$, L. J. Suva, C. O. Montgomery

Department of Orthopaedics, University of Arkansas for Medical Sciences, 4301 W Markham Street, Slot \# 341, Little Rock, AR 72205, USA

e-mail: smorell@uams.edu

J. M. Gardner

Department of Pathology, University of Arkansas for Medical

Sciences, Little Rock, AR, USA lymph node measuring $1.8 \mathrm{~cm}$ in diameter. A biopsy of this lymph node was negative for malignancy.

The patient's progressive bilateral leg pain was followed later by right ankle and right elbow pain. She also had pruritus but it was thought that her pruritus was related to her chronic autoimmune hepatitis and primary sclerosing cholangitis. She stated that her knee pain was dull and constant throughout the day and that she was unable to stand or walk for long periods owing to the pain. The patient had received transient relief of her knee pain with oral pain medications and intraarticular steroid injections. However, because of persistent and severe right knee pain, MRI of the knee was obtained, and based on the abnormal bone marrow findings, the patient was referred to orthopaedic oncology. Socially, the patient was a smoker, but no other relevant factors were noted, and there was no relevant family history.

On physical examination, numerous hyperpigmented erythematous macules and papules on the back, bilateral lower arms, thighs, and lower legs with surrounding excoriations were noted. No visible or palpable masses were found on examination of her knee. The patient's knee pain and tenderness did not localize to any particular portion of her knee on examination. Examination of her knee was significant for worsening pain with flexion and extension of the knee, but no ligamentous instability was noted. There was no warmth or redness noted on examination. The patient was otherwise neurovascularly intact. On examination of her abdomen, she had hepatosplenomegaly. Laboratory studies including complete blood count and C-reactive protein were normal.

Radiographic evaluation of both knees (Fig. 1), MRI of the right knee (Fig. 2), and a whole-body scan (Fig. 3) were obtained. 
Based on the history, physical examination, laboratory studies, and imaging studies, what is the differential diagnosis at this point?

\section{Imaging Interpretation}

The patient's bilateral knee plain radiograph showed no abnormalities (Fig. 1). The sagittal MR image of the right knee (Fig. 2) with contrast showed diffuse heterogeneous marrow signal intensity. The MR image without contrast

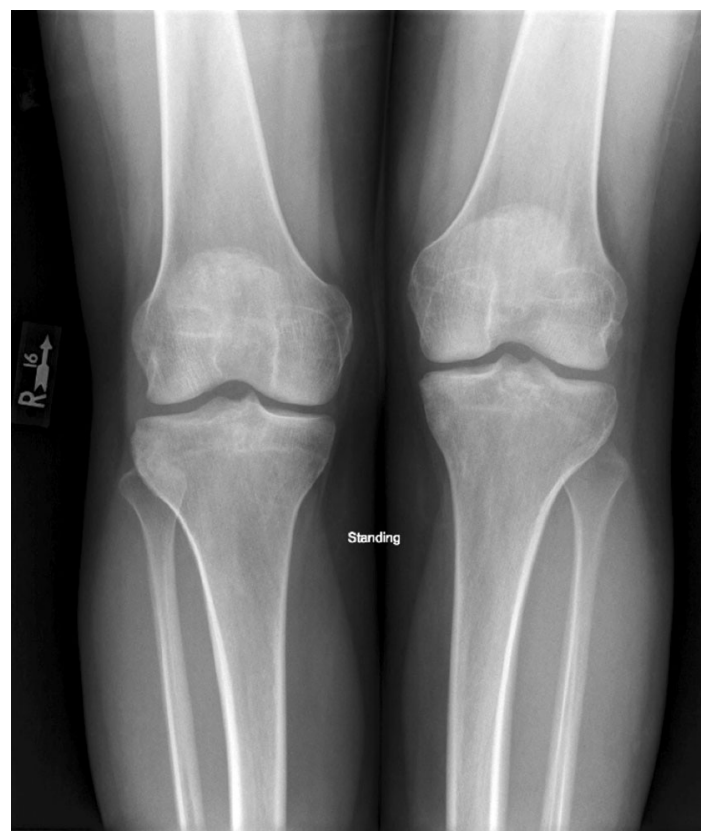

Fig. 1 A bilateral knee radiograph was obtained early on and was read as "normal".

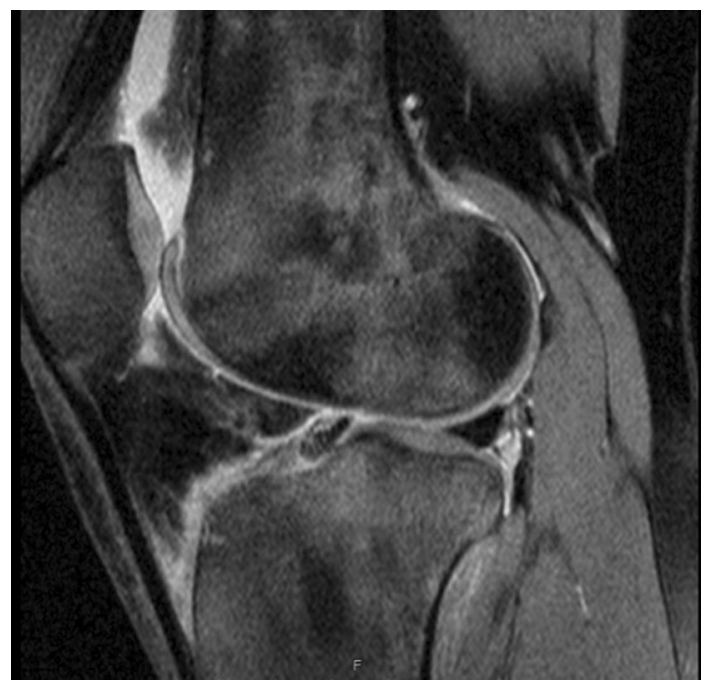

Fig. 2 A sagittal MR image of the right knee with contrast shows diffuse heterogeneous marrow signal intensity. also showed diffuse heterogeneous marrow signal intensity. There were patchy areas of diminished signal intensity on the T2-weighted images, and increased signal intensity on the fat-saturated sequences. No cortical invasion or disruption was apparent. The knee ligaments were intact.

A whole-body bone scan (Fig. 3) showed homogeneous symmetric diffuse increased radiotracer uptake along the bilateral proximal and distal femurs, proximal tibias, and calcanei. Because of the imaging findings, a bone biopsy of the right distal femur was performed.

\section{Differential Diagnosis}

Lymphoma with Secondary Bone Involvement (Stage IV)

SAPHO syndrome (previously known as chronic recurrent multifocal osteomyelitis, but more recently renamed to reflect the features that often accompany it, specifically synovitis, acne, pustulosis, hyperostosis, and osteitis [11])

\section{Metastatic Carcinoma}

\section{Systemic Mastocytosis}

Based on the history, physical examination, laboratory studies, imaging studies, and histologic picture, what is the diagnosis and how should the patient be treated?

\section{Histology Interpretation}

Aggregates of spindled mast cells were present in the marrow space on the bone biopsy specimen. Normal trilineage hematopoietic bone marrow elements also were present (Fig. 4). Spindled mast cells were arranged as peritrabecular aggregates and scattered single cells were seen in the marrow space on the bone biopsy specimen. These mast cells showed strong expression of mast cell tryptase (brown staining) by immunohistochemistry (Fig. 5).

\section{Diagnosis}

Systemic mastocytosis.

\section{Discussion and Treatment}

The WHO classifies mastocytosis as a myeloproliferative neoplasm derived from a clonal proliferation of mast cells 


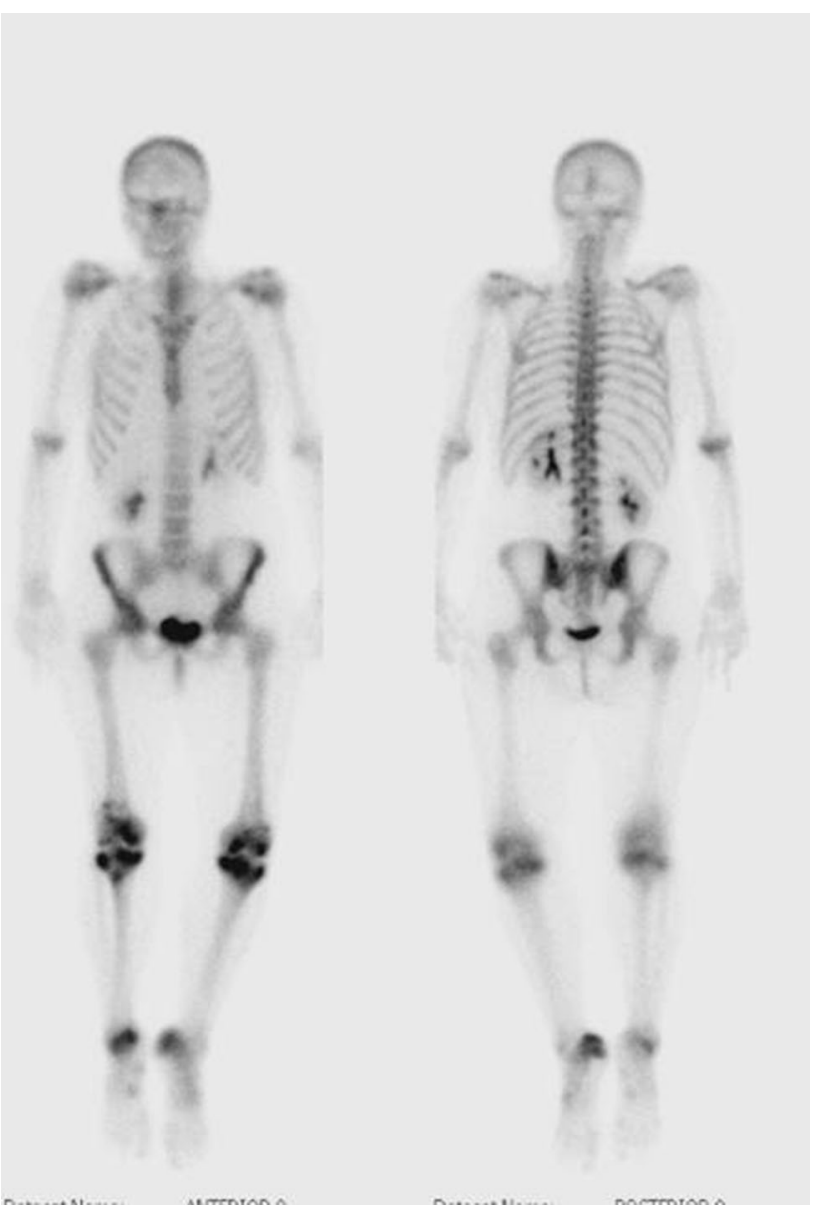

Fig. 3 A whole-body bone scan shows homogeneous symmetric diffuse increased radiotracer uptake along the bilateral proximal and distal femurs, proximal tibias, and calcanei.

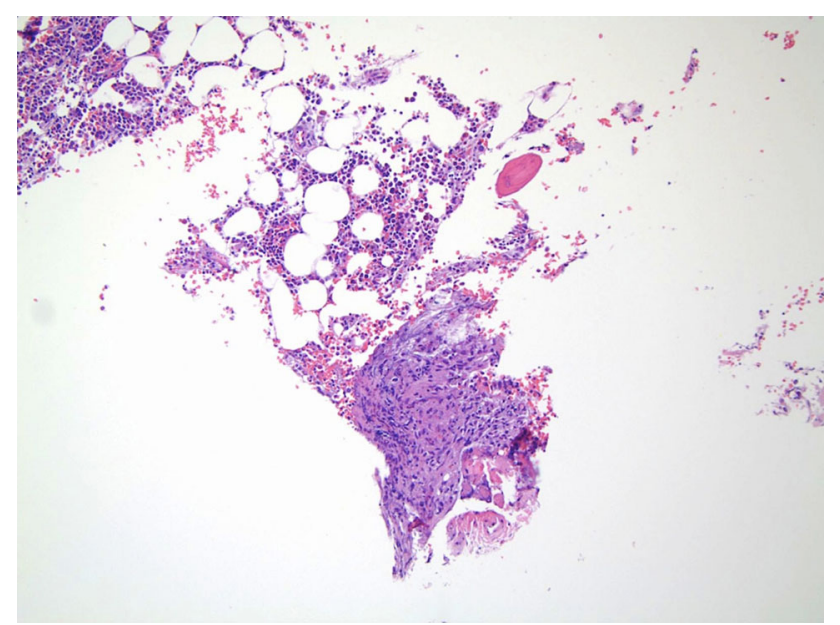

Fig. 4 Aggregates of spindled mast cells (center) are present in the marrow space on the bone biopsy specimen. Normal trilineage hematopoietic bone marrow elements also are present (upper left) (Stain, hematoxylin \& eosin; original magnification, $\times 200$ ). (Published with permission from Matthew R. Lindberg MD, Department of Pathology, University of Arkansas for Medical Sciences, Little Rock, AR, USA.)

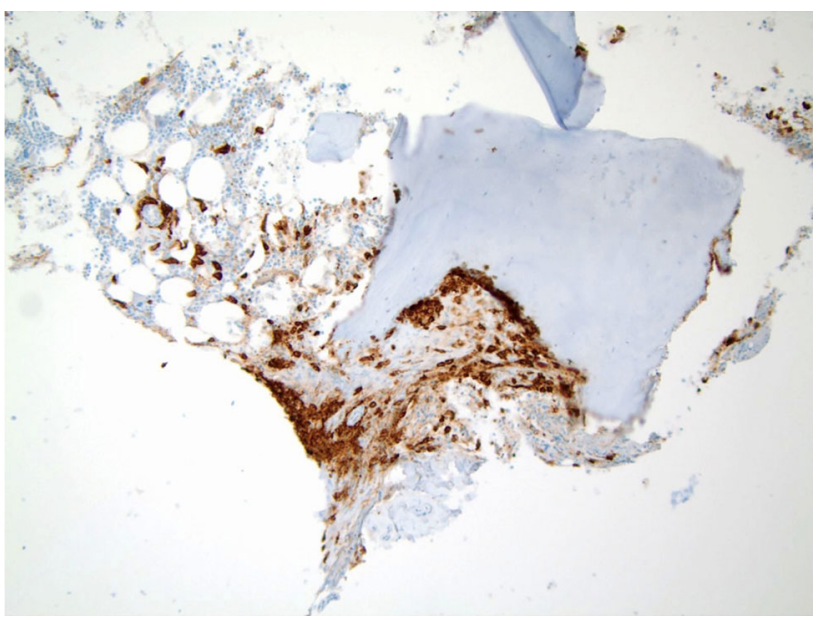

Fig. 5 Spindled mast cells are arranged as peritrabecular aggregates and scattered single cells in the marrow space on bone biopsy specimen. These mast cells show strong expression of mast cell tryptase (brown staining) by immunohistochemistry (Stain, mast cell tryptase immunohistochemistry; original magnification, $\times 200$ ). (Published with permission from Matthew R. Lindberg MD, Department of Pathology, University of Arkansas for Medical Sciences, Little Rock, AR, USA.)

or its precursors [13, 17]. The myriad of symptoms the patient experienced are characteristic of systemic mastocytosis. This is a very rare disease and the exact incidence is unknown. It occurs in men and women equally. The childhood form of the disease usually only involves the skin [9].

Systemic mastocytosis can present with multiple symptoms involving various organ systems including skin (pruritus, hives, and flushing), gastrointestinal (pain, nausea, and vomiting), cardiovascular (palpitations and syncope), neurologic (cognitive difficulties, depression, and sleep disturbance), skeletal (pain, osteopenia, and fractures), and constitutional (fatigue, weakness, and chills) $[13,17]$. The patient's long history of pruritus initially was presumed to be the result of her hepatobiliary disease, but in retrospect it most likely was caused by her systemic mastocytosis for which pruritus is a very common symptom $[9,13,14]$. The dermatologic findings at presentation were consistent with excoriations owing to diffuse pruritus $[4,13,14]$. The patient's chronic gastrointestinal symptoms with elevated liver enzymes also are consistent with systemic mastocytosis [14]. In addition, our patient had bone involvement, which is common in systemic mastocytosis [12]; bone involvement by mastocytosis can lead to pathologic fractures in some cases [12]. The osseous radiographic findings of systemic mastocytosis are variable: some cases are occult and not visible by imaging whereas others may display blastic lesions, lytic lesions, or a combination of both $[1,5,12]$. Patients with systemic mastocytosis can go undiagnosed for many years given the 
nonspecific nature of the symptoms, as was the case for our patient $[4,14,17]$.

Systemic mastocytosis is characterized by a monoclonal proliferation of atypical mast cells resulting from activating somatic point mutations of the $c$-kit proto-oncogene. These mutations usually involve exon 17 of $c$-kit with the imatinib-resistant mutation D816V being most frequent [6]. According to the WHO, systemic mastocytosis can be divided into several subtypes including, (1) indolent systemic mastocytosis; (2) systemic mastocytosis with associated clonal hematologic nonmast cell lineage disease; (3) mast cell leukemia; (4) mast cell sarcoma; and (5) aggressive systemic mastocytosis [4]. The major criterion for systemic mastocytosis is the presence of multifocal dense infiltrates of mast cells in the bone marrow; this finding has a $100 \%$ specificity, $100 \%$ positive predictive value, and $69 \%$ sensitivity for the presence of systemic mastocytosis [4]. Tryptase immunohistochemical staining is a highly sensitive marker for mast cells and can be used to help highlight mast cell infiltrates on biopsy $[6,12]$. Additional minor criteria for the diagnosis of systemic mastocytosis include detection of the $c$-kit mutation, organ system involvement other than skin, serum tryptase levels greater than $20 \mu \mathrm{g} / \mathrm{L}$, and extracutaneous mast cells with $\mathrm{CD} 2+$ and/or CD25+ expression. The WHO criteria for the diagnosis of systemic mastocytosis states one must have one major criteria finding and one minor finding or three minor findings to have the diagnosis of systemic mastocytosis $[4,9,12]$.

The leading diagnosis at presentation given the patient's history of lymphadenopathy was nodal lymphoma with secondary bone involvement. Lymphoma is a malignancy composed of clonal populations of either B or T lymphocytes or related hematopoietic cells [2, 8]. Lymphoma most commonly arises in lymph nodes, but it also may arise as a primary process in extranodal sites, including bone $[2,3]$. Additionally, primary nodal lymphomas may secondarily spread to bone and other extranodal sites $[2,3,8]$. Thus, lymphoma was an initial major differential diagnosis for the diffuse osseous involvement seen on MR images and whole-body scan in our patient $[2,8,15]$. The patient's diffuse pain and malaise, although nonspecific, are not unusual presenting symptoms of lymphoma [2, 3]. The patient's pruritus also could have been paraneoplastic, a situation that previously has been associated with lymphoma [19]. Osseous involvement by lymphoma can range from marrow-replacing lesions only to destructive lytic lesions with soft tissue extension [3, 18]. Biopsy with histopathologic and/or flow cytometric evaluation is the gold standard for diagnosis or exclusion of lymphoma. Biopsy of lymphoma involving bone typically would show aggregates or sheets of clonal $\mathrm{B}$ or $\mathrm{T}$ lymphocytes with varying degrees of cytologic atypia (depending on the subtype of lymphoma). In contrast, the bone biopsy specimen in our patient showed aggregates of atypical mast cells instead of aggregates or sheets of lymphocytes, findings that excluded a diagnosis of lymphoma and confirmed a diagnosis of mastocytosis.

As noted earlier, SAPHO syndrome, consisting of synovitis, acne, pustulosis, hyperostosis, and osteitis, is an inflammatory bone condition that often involves skin and has some overlapping features with spondyloarthropathies [11]. This rare condition often is unrecognized because of the wide variability in its musculoskeletal and cutaneous manifestations [11]. This condition has been described in the literature as chronic recurrent multifocal osteomyelitis (CRMO) and various other names [7, 11]. In this case, given the patient's diffuse bone involvement and robust dermatologic findings, SAPHO syndrome was considered. Dermatologic findings of SAPHO syndrome appear as severe acne or pustules that most commonly present on the palms or soles [7, 11]. However, our patient had excoriations secondary to pruritus but lacked acneiform lesions or palmoplantar pustulosis as typically would be seen with SAPHO syndrome. Additionally, pruritus has not been a common reported symptom of SAPHO syndrome [7, 11]. Bone biopsy specimen in SAPHO syndrome often show remodeling and other reactive bone changes but lacks the aggregates of mast cells seen in our patient's bone biopsy specimen. The osseous radiographic presentation of patients with SAPHO typically ranges from osteolytic to osteosclerotic, but the osteosclerotic changes predominate with a thickened cortex and narrowing of the intramedullary canal [10].

The diffuse osseous involvement along with the patient's history of smoking also raised concern for metastatic bone disease [16]. In addition, chronic pruritus has been seen in some cases of carcinoma [19]. Cancer of unknown primary origin with metastatic bony involvement is a common occurrence. Typically, when a primary tumor is present, it can be found using routine diagnostic imaging. Our patient's initial imaging did not identify any primary tumor. Therefore, although metastatic carcinoma was still part of the differential diagnosis, the lack of an identifiable primary lesion and the relatively young age of the patient moved metastatic bone disease lower on our list of differential diagnoses [15]. As for other neoplastic diseases, the gold standard for diagnosis of metastatic bone disease is bone biopsy. On histologic examination, metastatic carcinoma in the bone typically would show nests or glands of atypical epithelioid cells infiltrating the marrow space with associated desmoplasia (reactive fibrosis) and reactive bone changes. Our patient's bone biopsy specimen lacked these findings and instead showed atypical mast cell aggregates.

Currently, there is no known cure for aggressive systemic mastocytosis. Thus, therapeutic goals are symptom 
reduction and quality of life improvement rather than cure. For aggressive systemic mastocytosis, as in our patient, cytoreductive therapies including cladribine, interferonapha $2 \mathrm{~b}$, glucocorticoids, hydroxyurea, and tyrosine kinase inhibitors have been suggested $[4,9]$.

Although we provided aggressive treatment, this patient has done poorly. She originally was treated before her diagnosis of aggressive systemic mastocytosis with multiple autoimmune suppressive therapies including mesalamine, azathioprine, and infliximab for her cholangitis along with Crohn's disease. She was treated with Atarax ${ }^{\circledR}$ (Pfizer Inc, Peapack, NJ, USA) along with cholestyramine for pruritus symptoms. Her condition continued to worsen, and she eventually was prescribed interferon therapy after her diagnosis. She continued to deteriorate and was prescribed nilotinib (tyrosine kinase inhibitor). She currently receives only certolizumab for Crohn's disease. She has continued to deteriorate and is receiving home health hospice care.

\section{References}

1. Barer M, Peterson LF, Dahlin DC, Winkelmann RK, Stewart JR. Mastocytosis with osseous lesions resembling metastatic malignant lesions in bone. J Bone Joint Surg Am. 1968;50:142-152.

2. Bremnes RM, Bremnes Y, Donnem T. High-grade non-Hodgkin's lymphoma treated in northern Norway: treatment, outcome, and prognostic factors. Acta Oncol. 1999;38:117-124.

3. Demircay E, Hornicek FJ Jr, Mankin HJ, Degroot H 3rd. Malignant lymphoma of bone: a review of 119 patients. Clin Orthop Relat Res. 2013;471:2684-2690.

4. Fernandes IC, Teixeira Mdos A, Freitas I, Selores M, Alves R, Lima M. Adult mastocytosis: a review of the Santo Antonio Hospital 's experience and an evaluation of World Health Organization criteria for the diagnosis of systemic disease. An Bras Dermatol. 2014;89:59-66.

5. Hills E, Dunstan CR, Evans RA. Bone metabolism in systemic mastocytosis: a case report. J Bone Joint Surg Am. 1981;63:665-669.
6. Horny HP, Sotlar K, Valent P. Mastocytosis: state of the art. Pathobiology. 2007;74:121-132.

7. Kundu BK, Naik AK, Bhargava S, Srivastava D. Diagnosing the SAPHO syndrome: a report of three cases and review of literature. Clin Rheumatol. 2013;32:1237-1243.

8. Levis A, Pietrasanta D, Godio L, Vitolo U, Ciravegna G, Di Vito F, Gavarotti P, Guglielmelli T, Orsucci L, Raviolo E, Rota Scalabrini D, Salvi F, Tonso A, Aglietta M, Boccadoro M, Gallamini A, Saglio G, Scassa E, Gallo E. A large-scale study of bone marrow involvement in patients with Hodgkin's lymphoma. Clin Lymphoma. 2004;5:50-55.

9. Llado AC, Mihon CE, Silva M, Galzerano A. Systemic mastocytosis: a diagnostic challenge. Rev Bras Hematol Hemoter. 2014;36:226-229.

10. Mann B, Shaerf DA, Sheeraz A, Skinner JA, Saifuddin A. SAPHO syndrome presenting as widespread bony metastatic disease of unknown origin. Rheumatol Int. 2012;32:505-507.

11. Nguyen MT, Borchers A, Selmi C, Naguwa SM, Cheema G, Gershwin ME. The SAPHO syndrome. Semin Arthritis Rheum. 2012;42:254-265.

12. Ozdemir D, Dagdelen S, Erbas T. Systemic mastocytosis. Am J Med Sci. 2011;342:409-415.

13. Pardanani A. Systemic mastocytosis in adults: 2012 update on diagnosis, risk stratification, and management. Am J Hematol. 2012;87:401-411.

14. Pardanani A, Tefferi A. Systemic mastocytosis in adults: a review on prognosis and treatment based on 342 Mayo Clinic patients and current literature. Curr Opin Hematol. 2010;17: $125-132$.

15. Ponzoni M, Ciceri F, Crocchiolo R, Famoso G, Doglioni C. Isolated bone marrow occurrence of classic Hodgkin's lymphoma in an HIV-negative patient. Haematologica. 2006;91:ECR04.

16. Varadhachary GR, Raber MN. Cancer of unknown primary site. $N$ Engl J Med. 2014;371:757-765.

17. Vardiman JW, Thiele J, Arber DA, Brunning RD, Borowitz MJ, Porwit A, Harris NL, Le Beau MM, Hellstrom-Lindberg E, Tefferi A, Bloomfield CD. The 2008 revision of the World Health Organization (WHO) classification of myeloid neoplasms and acute leukemia: rationale and important changes. Blood. 2009;114:937-951.

18. Weber KL. Evaluation of the adult patient (aged $>40$ years) with a destructive bone lesion. J Am Acad Orthop Surg. 2010; 18:169-179.

19. Yosipovitch G. Chronic pruritus: a paraneoplastic sign. Dermatol Ther. 2010;23:590-596. 